\title{
Exome Sequencing Identifies Compound Heterozygous Mutations in SCN5A Associated with Congenital Complete Heart Block in the Thai Population
}

\author{
Chuphong Thongnak, ${ }^{1,2,3}$ Pornprot Limprasert, ${ }^{4}$ \\ Duangkamol Tangviriyapaiboon, ${ }^{5}$ Suchaya Silvilairat, ${ }^{6}$ Apichaya Puangpetch, ${ }^{1,2}$ \\ Ekawat Pasomsub, $^{3,7}$ Chonlaphat Sukasem, ${ }^{1,2}$ and Wasun Chantratita ${ }^{3,7}$ \\ ${ }^{1}$ Division of Pharmacogenomics and Personalized Medicine, Department of Pathology, Faculty of Medicine Ramathibodi Hospital, \\ Mahidol University, Bangkok, Thailand \\ ${ }^{2}$ Laboratory for Pharmacogenomics, Somdech Phra Debaratana Medical Center (SDMC), Ramathibodi Hospital, Bangkok, Thailand \\ ${ }^{3}$ Excelence Center for Genomic Medicine, Faculty of Medicine Ramathibodi Hospital, Mahidol University, Bangkok, Thailand \\ ${ }^{4}$ Human Genetics Unit, Department of Pathology, Faculty of Medicine, Prince of Songkla Univerisity, Hat Yai, Songkhla, Thailand \\ ${ }^{5}$ Rajanagarindra Institute of Child Development, Chiang Mai, Thailand \\ ${ }^{6}$ Division of Pediatric Cardiology, Department of Pediatrics, Faculty of Medicine, Chiang Mai University, Chiang Mai, Thailand \\ ${ }^{7}$ Virology Laboratory, Department of Pathology, Faculty of Medicine Ramathibodi Hospital, Mahidol University, Bangkok, Thailand
}

Correspondence should be addressed to Chonlaphat Sukasem; chonlaphat.suk@mahidol.ac.th and Wasun Chantratita; wasun.cha@mahidol.ac.th

Received 12 July 2016; Accepted 18 October 2016

Academic Editor: Marco E. M. Peluso

Copyright (C) 2016 Chuphong Thongnak et al. This is an open access article distributed under the Creative Commons Attribution License, which permits unrestricted use, distribution, and reproduction in any medium, provided the original work is properly cited.

Background. Congenital heart block is characterized by blockage of electrical impulses from the atrioventricular node (AV node) to the ventricles. This blockage can be caused by ion channel impairment that is the result of genetic variation. This study aimed to investigate the possible causative variants in a Thai family with complete heart block by using whole exome sequencing. Methods. Genomic DNA was collected from a family consisting of five family members in three generations in which one of three children in generation III had complete heart block. Whole exome sequencing was performed on one complete heart block affected child and one unaffected sibling. Bioinformatics was used to identify annotated and filtered variants. Candidate variants were validated and the segregation analysis of other family members was performed. Results. This study identified compound heterozygous variants, c.101G > A and c.3832G > A, in the SCN5A gene and c.28730C > T in the TTN gene. Conclusions. Compound heterozygous variants in the SCN5A gene were found in the complete heart block affected child but these two variants were found only in the this affected sibling and were not found in other unaffected family members. Hence, these variants in the SCN5A gene were the most possible disease-causing variants in this family.

\section{Introduction}

Congenital heart block is an uncommon disorder that occurs in about 1 in 20,000 live births [1]. It is characterized by anatomical or functional impairment in the conduction system which is caused by blockage of electrical impulses from atrioventricular node (AV node) to the ventricles [2]. The severity of heart block ranges from first-degree in which electrical impulse to the AV node is slower than normal to third-degree or complete heart block in which electrical impulses from the atrium do not reach ventricles at all [3].

The conduction defect can be caused by a defective link between cardiomyocytes or by ion channel impairment that changes action potential shapes [4]. Inherited defects in cardiac conduction have been linked to genetic variants in several genes such as SCN5A, SCN1B, KCNJ2, HCN4, 

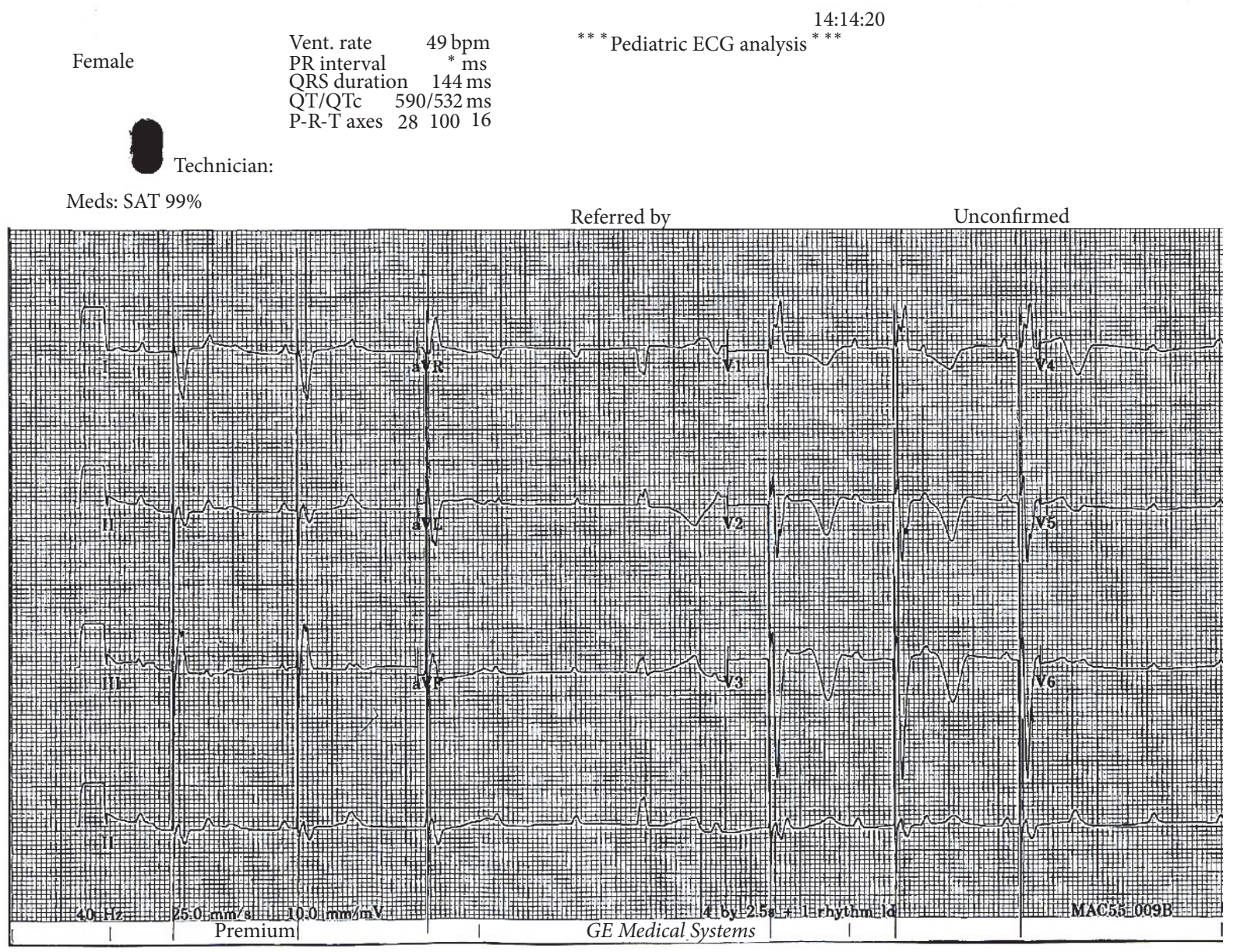

FIGURE 1: Electrocardiography of index case (III-2) shows third-degree atrioventricular block, atrial rate 100/min, ventricular rate 49/min, and ventricular pacing spikes before some QRS complexes.

NKX2-5, TBX5, LMNA, and ANKB [4-9]. Among these genes, $S C N 5 A$ has been frequently reported with various phenotypes [10]. SCN5A encodes $\alpha$ subunit of the cardiac sodium channel (NaV1.5) which controls the flow of sodium ions into cells that is essential in generation and transmission of electrical impulses [11]. A nonfunctional protein, which is caused by a mutation in the $S C N 5 A$ gene, reduces entrance of sodium into the cells that results in difficulty producing and transmitting electrical signals resulting in heart block [12].

Mutations in SCN5A, which lead to loss or gain of sodium channel function, are associated with a spectrum of cardiac diseases including Brugada syndrome, Long QT syndrome type 3 , sick sinus syndrome, and progressive familial heart block [12-16].

With the advantages of next-generation sequencing especially whole exome sequencing that can explore the sequence of all exons in a single experiment, sequencing has been used in several studies for comprehensive and unbiased identification of causative variants of diseases in the last decade $[17,18]$. Likewise, whole exome sequencing has been used in other cardiovascular related studies to identify diseasecausing mutations of familial atrial septal defects [19].
This study aims to investigate the possible causative variants in a Thai family with complete heart block by using whole exome sequencing. A combined method of familial data, exome sequencing, bioinformatics, and segregation analysis was able to identify 6 variants in 5 genes in which 2 variants in SCN5A were the most plausible disease-causing variants for heart block.

\section{Materials and Methods}

2.1. Subjects. The family in this study had 3 generations from which blood samples for DNA preparations were collected: grandmother (I-2), mother (II-2), and two children (III-2 and III-3). The II-2 was a single mother; therefore, we could not obtain blood for DNA from the father. The index case (III2) had a third-degree AV block and had undergone cardiac surgery for epicardial pacemaker implantation at the age of 18 months. She was diagnosed with autism according to the DSM-IV at the age of 6 years. The other two siblings had no heart defects but one had learning disability (III1, no DNA) and the other was autistic (III-3) (Figure 1). 
Extracted DNA samples from III-2 and III-3 were controlled for their quality by measuring DNA concentration using Nanodrop ND1000 and measuring fragmentation of DNA by agarose gel electrophoresis. These 2 samples were prepared for whole exome sequencing. DNA samples from other family members (I-2, II-2) were prepared for segregation analysis. Written informed consent was obtained from adult family members themselves and for all children and all procedures were approved by the Institutional Review Board (MURA2012/02/SN1) of the Faculty of Medicine Ramathibodi Hospital, Mahidol University, and EC48/364-006-3 of the Faculty of Medicine, Prince of Songkla University.

\subsection{Exome Sequencing and Data Analysis. Whole exome se-} quencing was performed on family members III-2 and III-3. Samples were prepared following standard SOLID 5500xl (Applied Biosystems, California, USA) protocols for whole exome sequencing. Three micrograms of genomic DNA of each sample were fragmented using Covaris S2 (Covaris, Massachusetts, USA) and were captured for exome sequencing using TargetSeq Exome and Custom Enrichment System (Invitrogen, California, USA). The captured DNA was sequenced with $150 \mathrm{bp}$ paired-end read on the SOLID $5500 x \mathrm{l}$ system according to the manufacturer's protocol. Primary analysis was performed on the sequencing machine using SOLID ICS software; then, raw sequenced data was transferred to the LifeScope Genomic Analysis server for secondary analysis. Sequence reads were mapped to the human reference genome assembly hg19 (GRCh37); then, variants calling for SNV, insertions, and deletions were performed.

Tertiary analysis was performed by using Golden Helix SVS software on identified candidate variants. Variants were filtered for minimum genotype quality of 20 and minimum coverage depths of 10 and then the qualified variants were annotated with the UCSC KnownGenes database to remove noncoding and synonymous variants. High frequency variants (minor allele frequencies greater than $2 \%$ ) were excluded by annotation with allele frequencies from the 1000 Genomes Project Phase 3 [20] and an in-house exome database which consists of 172 Thai individuals. Deleterious protein functions were predicted using dbNSFP that compiled prediction scores from eleven prediction algorithms (SIFT, Polyphen2, LRT, MutationTaster, MutationAssessor, FATHMM, VEST3, CADD, MetaLR, MetaSVM, and PROVEAN) and 4 conservation scores, PhyloP, phastCons, GERP++, and SiPhy, and other related information [21]. Variants that were predicted not to alter protein function by any algorithm were excluded in this step. To narrow down variants, variants were focused on where these were located in the candidate genes list (Table 1). This genes list was created from combined known and suspected genes involved in the cardiovascular system from several sources and the cardiovascular defective candidate genes from the Enlis Genome Research software (Enlis, Berkeley, CA) gene panel. This genes list consists of 359 genes in total. Lastly, variants were filtered by their genotype. A genotype that was only in family member III-2 was indicated as a candidate variant for heart block. The summarized variants filter steps are shown in Figure 3.

Additionally, pathogenic variants which were related to other diseases followed were explored in The American College of Medical Genetics and Genomics (ACMG) recommendations for reporting of incidental findings in clinical exome and genome sequencing [22]. Known pathogenic variants were identified by using The Human Gene Mutation Database (HGMD) [23].

2.3. Variant Validations and Segregation Analysis. Sanger sequencing was used to validate the candidate variants found in whole exome sequencing and segregation analyses were performed on the family members. Primers were designed using the Primer3 version 0.4.0 web-based tool [24]. Sequencing reactions were performed using Applied Biosystems 3130 DNA Analyzer (Life Technologies, Carlsbad, CA, USA).

\section{Results}

A family with complete heart block in only one of the 3rd generation family members was explored. By whole exome sequencing, a total of 99,834 variants in family members III-2 and III- 3 were detected with an average depth over $60 \mathrm{x}$ coverage. After removal of low quality, noncoding and synonymous variants, 14,284 variants remained. Subsequently, variants were reduced by a filtering pipeline that included variants with minor allele frequencies, inheritance models, and a candidate gene list which reduced the number of variants to 36 variants in 28 genes. Finally, variants were prioritized and selected as candidate variants by annotated information from the deleterious protein function prediction database that resulted in 21 heart defective candidate variants in 18 genes. A list of candidate variants is shown in Table 2 . All 21 candidate variants were then investigated for validation and segregation analysis. Six variants successfully passed this step while the other variants were dropped for 2 reasons: (1) a discordance between whole exome sequencing and Sanger sequencing or (2) variants found in the affected child being found in other unaffected family members except for two or more variants that were found in the same gene which indicated compound heterozygous inheritance. These 6 variants in 5 genes were identified as candidate variants for heart defects in this family. The list of final candidate variants is shown in Table 3.

Two nonsynonymous missense variants in the SCN5A gene in this study, c.101G >A and c.3832G > A (NM_000335), were likely to be present in a compound heterozygous fashion because 2 heterozygous variants were found in same gene in the affected case but only one or none of them were found in unaffected family members. Heterozygous c.101G $>$ A was found in III-2 (index) and II-2 (mother) while heterozygous c.3832G $>$ A was found in III-2 (index) and III-3 (brother) (Figure 2). Chromatograms of both variants in all available subjects are shown in Figure 4.

All other 4 variants were nonsynonymous missense variants in which their genotypes were heterozygous. These variants consisted of c.28730C $>\mathrm{T}$ (NM_133378) in the TTN gene, c.449C>T (NM_153240) in the NPHP3 gene, 


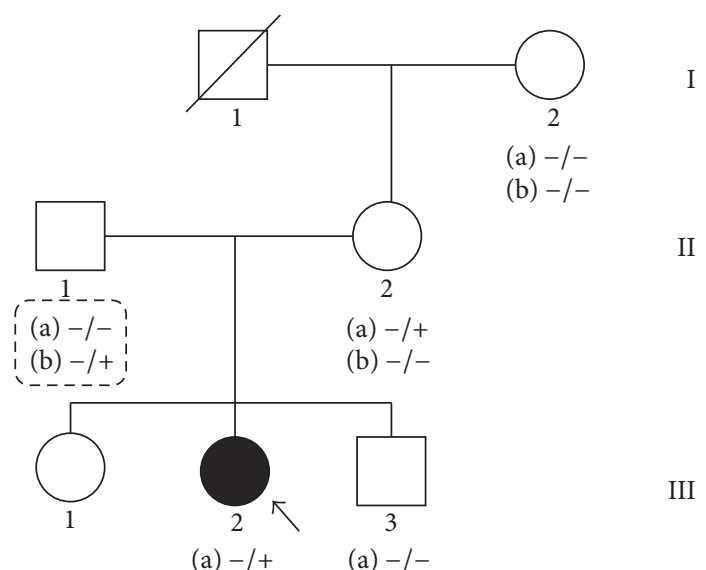
(a) $-1+$
(a) $-1-$
(b) $-1+$
(b) $-1+$

FIGURE 2: The lineage of the family with complete heart block indicated by the dark symbol, females by circles, and males by squares. Letters (a) and (b) indicate genotypes of 2 variants in the SCN5A gene for c.101G $>$ A and c.3832G $>$ A. The homozygous reference genotypes are indicated by $(-/-)$ and heterozygous alternate genotypes by $(-/+)$. The genotypes of II-1 are presumed genotypes which are inferred from his children.

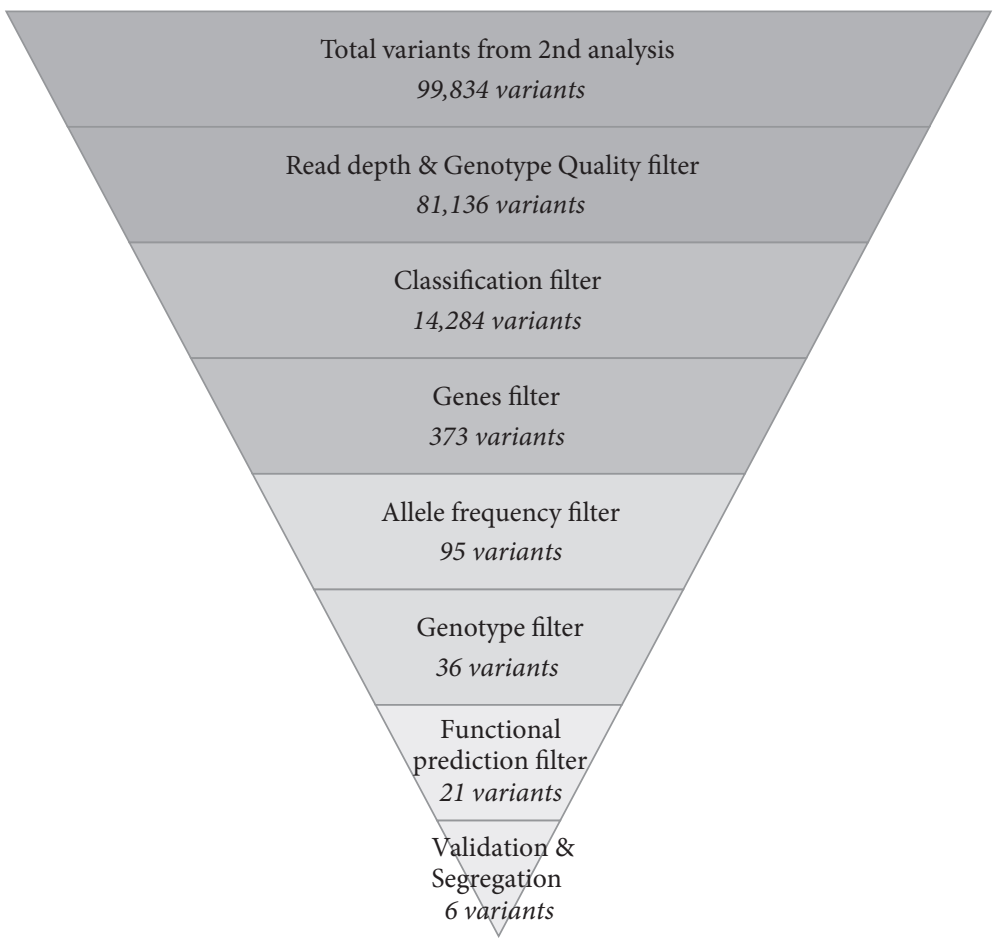

FIGURE 3: Filtering procedure of variants obtained by whole exome sequencing 2nd data analysis.

c.1133A > T (NM_012144) in the DNAI1 gene, and c.13481A >C (NM_002458) in the MUC5B gene. TTN encoded Titin or connectin, a giant muscle protein, is expressed in the cardiac and skeletal muscles. NPHP3 encodes a protein that is required for normal ciliary development. DNAI1 encodes a member of the dynein intermediate chain family. Lastly, $M U C 5 B$ encodes a protein member of the mucin family.

Finally, incidental findings in the whole exome data in this family following ACMG recommendations were explored. Aside from variants in the SCN5A gene that were assigned in the ACMG panel as known/expected pathogenic variants for Romano-Ward Long QT syndromes Types 1, 2, and 3 and Brugada syndrome, which were key variants in this family, a variant in the $M Y B P C 3$ gene that was assigned in the ACMG panel for hypertrophic/dilated cardiomyopathy was explored and validated.

\section{Discussion}

Although a congenital heart defect was found in this autistic patient, this was unlike the Timothy syndrome which is a rare disorder that affects heart, nervous system, and fingers/toes. 

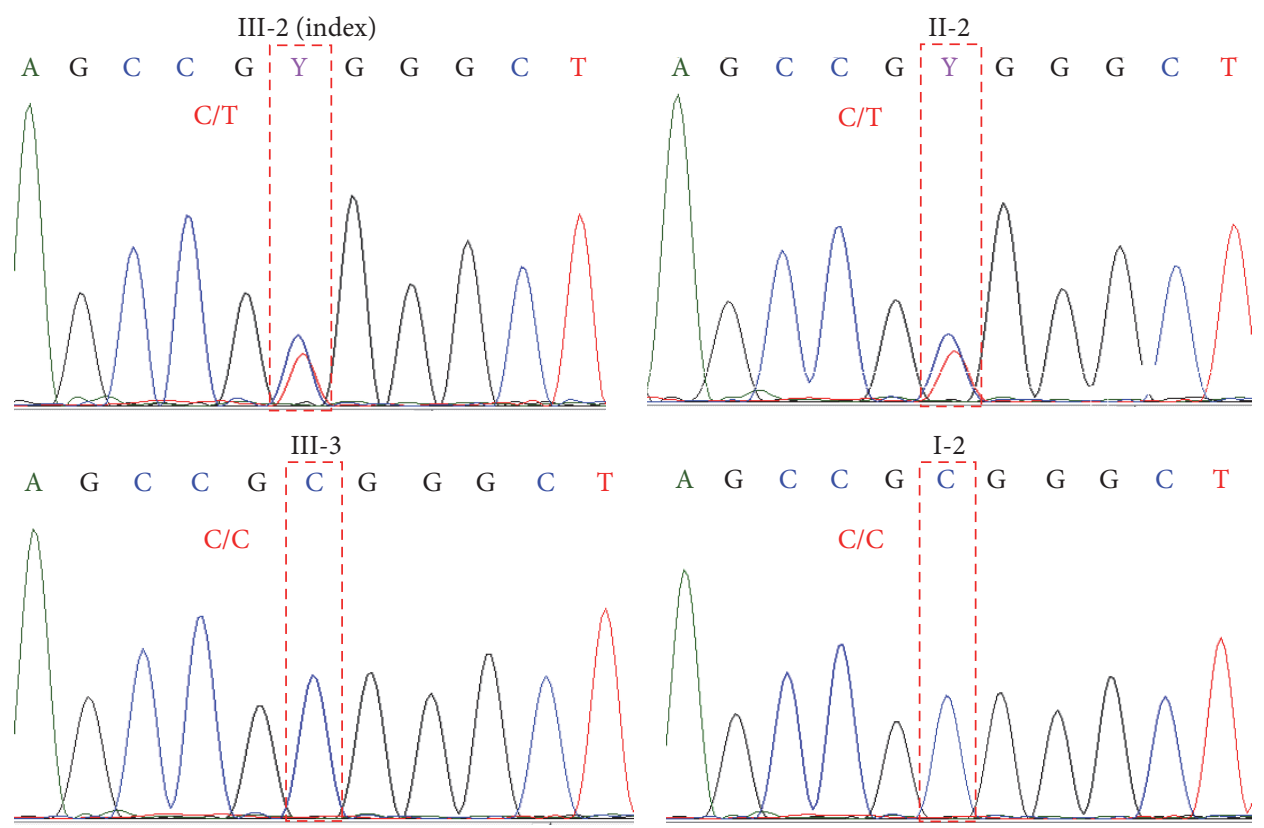

(a) SCN5A c.101G >A [C>T]
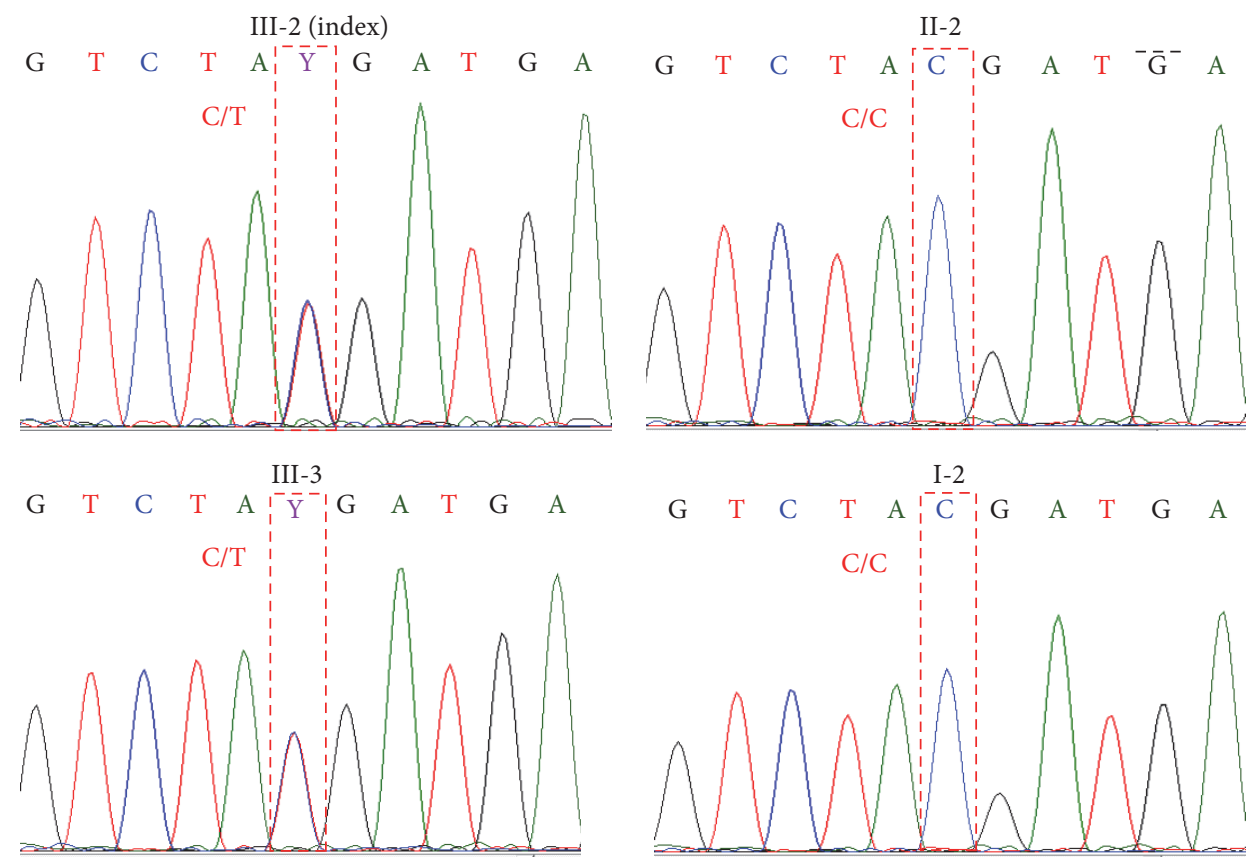

(b) SCN5A c.3832G $>$ A $[\mathrm{C}>\mathrm{T}]$

FIGURE 4: Chromatograms of 2 heterozygous missense variants in SCN5A gene: c.101G>A (a) and c.3832G>A (b). Letters in [] indicate complementary (FWD) alleles.

Syndactyly, the webbing of fingers and toes, one of Timothy syndrome signs, was not found in this case. Moreover, by direct sequencing and whole exome sequencing, variants in the CACNA1C gene, which are the common causes of both classical and atypical Timothy syndrome, were not detected [25]. Therefore, it was inferred that heart block and autism in this family are a coincidence.

SCN5A, a cardiac sodium channel gene, is important in generation and transmission of electrical impulses by its role in controlling the flow of sodium ions into cells [11]. Several variants in the SCN5A that are located in four homologous domains of alpha subunit of $\mathrm{NaV1.5}$, including 6 segments of each domain and linkers, N-terminal, and C-terminal, have been reported to be associated with various phenotypes of cardiac diseases [26]. The mechanism of SCN5A mutations associated with cardiac conduction disease has been explained by loss of function in NaV1.5 channels. Loss of $\mathrm{NaV} 1.5$ function leads to a reduction of inward sodium flow in 
TABLE 1: List of genes involved in cardiovascular system defects.

\begin{tabular}{|c|c|c|c|c|c|c|c|c|c|}
\hline$A A R S 2$ & ATP6V0A2 & CSRP3 & FASTKD2 & HAND1 & $L M F 1$ & MYH11 & PLN & SFTPA1 & TGFB2 \\
\hline$A B C A 1$ & $A T R X$ & CTF1 & FBLN5 & HAND2 & LMNA & MYH6 & PLOD1 & SFTPA2 & TGFB3 \\
\hline$A B C A 3$ & B3GAT3 & CTNNA3 & $F B N 1$ & HCN4 & LPIN1 & MYH7 & PNPLA3 & SFTPB & TGFBR1 \\
\hline ABCC6 & $B A G 3$ & DES & FBN2 & $H E R G$ & $L P L$ & MYL2 & PPARA & SFTPC & TGFBR2 \\
\hline ABCC 9 & $B C O R$ & DHCR24 & FGD1 & HFE & LRP5 & MYL3 & PPARG & SFTPD & THEMIS \\
\hline ABCG5 & $B M P R 2$ & DHCR7 & FGFR2 & HOXA1 & LTBP4 & MYLK & PPP1R17 & $S G C D$ & TLL1 \\
\hline ABCG8 & $B R A F$ & $D L L 3$ & FHL1 & HRAS & $M A P 2 K 1$ & MYLK2 & PRDM16 & $S G C G$ & TMEM43 \\
\hline$A C A D V L$ & CACNA1B & $D M D$ & FHL2 & $I G B P 1$ & $M A P 2 K 2$ & MYO6 & PRKAB2 & SHOC2 & TMEM70 \\
\hline ACTA1 & CACNA1C & $D M P K$ & FKTN & $I L K$ & MED12 & MYOCD & PRKAG2 & SKI & TMPO \\
\hline ACTA2 & CACNA1D & DNAH11 & FLNA & $\operatorname{IRX} 4$ & $M E F 2 C$ & MYOM1 & PRKAR1A & SLC25A3 & TNNC1 \\
\hline ACTC & CACNA2D1 & DNAH5 & FLNB & $J A G 1$ & MHY11 & MYOT & PRKG1 & SLC25A4 & TNNI3 \\
\hline ACTC1 & CACNB2 & DNAI1 & FOXC2 & $\mathrm{JPH} 2$ & MIB1 & MYOZ2 & PSEN1 & SLC2A10 & TNNT2 \\
\hline ACTN2 & $C A L M 1$ & DNAJC19 & FOXH1 & $J U P$ & MID1 & $M Y P N$ & PSEN2 & SLMAP & $T N X B$ \\
\hline$A C V R 2 B$ & CALM2 & DNM1L & FOXRED1 & KCNA5 & $M K K S$ & $N E B L$ & PTPN11 & SMAD3 & ТОРВР1 \\
\hline$A C V R L 1$ & CALR3 & $D O L K$ & $F R Y L$ & KCND3 & $M K S 1$ & NEXN & $P T R F$ & SMAD4 & TPM1 \\
\hline$A D C K 3$ & CASQ2 & DPP6 & $F X N$ & KCNE1 & MOG1 & $N F 1$ & $R A F 1$ & SMAD9 & TPM2 \\
\hline$A D R B 1$ & CAV1 & DSC2 & $G A A$ & KCNE1L & MRPL3 & NIPBL & RAII & SNTA1 & TRDN \\
\hline$A G L$ & $C A V 3$ & DSG2 & GATA4 & KCNE2 & MTND1 & NKX2.5 & $R A N G R F$ & SNX3 & TRIM63 \\
\hline AKAP9 & $C B L$ & $D S P$ & GATA5 & KCNE3 & MTND5 & NKX2.6 & RASA1 & SOS1 & TRPM4 \\
\hline AKT3 & CBS & DTNA & GATA6 & $\mathrm{KCNH} 2$ & MTND6 & NKX2-5 & $R B M 10$ & SOX2 & TSFM \\
\hline ALMS1 & $C F C 1$ & EFEMP2 & GATAD1 & KCNJ2 & MTTD & NODAL & RBM20 & SOX7 & TTN \\
\hline$A L P K 3$ & CHD7 & $E I F 2 A K 4$ & GATT6 & KCNJ5 & MTTG & NOS1AP & $R E T$ & SPEG & $T T R$ \\
\hline ANGPTL3 & CHST14 & ELMOD2 & GDF1 & KCNJ8 & MTTH & NOTCH1 & RPL4 & SPRED1 & TWIST1 \\
\hline ANGPTL4 & COA5 & $E L N$ & GDF2 & KCNK3 & MTTI & $\mathrm{NOTCH} 2$ & $R P S A$ & SURF1 & TXNRD2 \\
\hline ANK2 & COL18A1 & $E M D$ & GJA1 & KCNQ1 & MTTK & NOTCH3 & $R Y R 1$ & SYNE1 & UQCRB \\
\hline ANKRD1 & COL1A1 & $E N G$ & GJA5 & KRAS & MTTL1 & $N P C 1$ & $R Y R 2$ & SYNE2 & USF1 \\
\hline ANO5 & COL1A2 & EPHX2 & GLA & $L A M A 4$ & MTTL2 & NPHP3 & SALL1 & $T A Z$ & $V C L$ \\
\hline APOA1 & $C O L 2 A 1$ & ESCO2 & $G L B 1$ & $L A M P 2$ & MTTM & $N P P A$ & SALL4 & $T B X 1$ & $V C P$ \\
\hline APOA2 & COL3A1 & $E V C$ & GLI3 & $L B R$ & MTTP & NRAS & $S C N 1 B$ & TBX20 & $V H L$ \\
\hline APOA5 & COL4A1 & EVC2 & GNAI2 & LCAT & MTTQ & NSDHL & $S C N 2 B$ & TBX3 & $X K$ \\
\hline$A P O B$ & COL5A1 & EYA1 & GPC3 & $L D B 3$ & MTTS1 & NUBPL & $S C N 3 B$ & TBX5 & ZASP \\
\hline APOC2 & COL5A2 & EYA4 & GPD1L & $L D L R$ & MTTS2 & РCSK9 & SCN4B & TCAP & ZFPM2 \\
\hline$A P O E$ & CREBBP & FANCA & GPIHBP1 & LDLRAP1 & $M U C 5 B$ & PDLIM3 & SCN5A & TCTN3 & ZIC3 \\
\hline ARHGAP31 & CRELD1 & FANCC & GSN & LEFTY2 & МYBРC3 & PEX7 & SCO2 & TERC & ZMPSTE24 \\
\hline$A R X$ & CRYAB & FANCD2 & $G U S B$ & LIPC & $M Y C N$ & PKP2 & SDHA & TERT & ZNF469 \\
\hline ATP5E & CSF2RA & FANCE & $H A D H$ & LIPI & MYF6 & PKP4 & SEMA5A & TFAP $2 B$ & \\
\hline
\end{tabular}

the cells that results in difficulty producing and transmitting electrical signals. Conduction defects, however, could occur apart from transmission of inward sodium. The mutations on SCN5A, p.R219H, p.R222Q, and p.R225W which express alternative pathways, through a cation leak through NaV1.5, have been reported to be associated with mixed arrhythmias and dilated cardiomyopathy $[27,28]$. In this study, 2 missense variants in the SCN5A gene in a patient who had complete heart block (III-2) were identified. The first variant, c.101G $>$ A, results in replacement of arginine by histidine (p.R34H) in exon 2 whereas the second variant, c.3832G $>A$, results in replacement of valine by isoleucine (p.V1278I) in exon 21. Both variants were rare variants in which alternate alleles were not found in the Asian population from 1000 genome databases and the Thai population from the Thai in-house exome database. Functional prediction results show that both variants were predicted to alter protein function which indicates that these variants have a high possibility to damage function of the sodium channel and cause the conduction defect in this case (Table 3).

c.101G $>$ A (p.R34H) is a novel variant which is located in the N-terminal cytoplasmic domain of the sodium channel alpha subunit (Figure 5). A variant (c.80G>A) in the same region, the $\mathrm{N}$-terminal cytoplasmic domain of sodium channel, has been reported to be associated with Brugada syndrome by Priori et al. [29]. Makita et al. have reported a mutation, which resulted in a stop codon (p.Q55X), presented in a Brugada syndrome affected patient with 1st-degree AV block history [30].

c.3832G $>$ A (p.V1278I) has been reported as a "disease causing mutation" of dilated cardiomyopathy in the HGMD database [31-33]. This variant is located in the S3 
TABLE 2: Candidate variants from whole exome sequencing.

\begin{tabular}{|c|c|c|c|c|c|}
\hline Position & Gene & Classification & Transcript & HGVS coding & $\mathrm{dbSNP}$ \\
\hline Chr1: 13036736 & PRAMEF22 & Nonsyn SNV & NM_001100631 & c. $808 \mathrm{~T}>\mathrm{A}$ & rs202011965 \\
\hline Chr2: 179402104 & $T T N$ & Nonsyn SNV & NM_003319 & c. $72635 \mathrm{G}>\mathrm{A}$ & - \\
\hline Chr2: 179542464 & TTN & Nonsyn SNV & NM_133378 & c. $30443 \mathrm{C}>\mathrm{T}$ & - \\
\hline Chr2: 179549988 & TTN & Nonsyn SNV & NM_133378 & c. $28730 \mathrm{C}>\mathrm{T}$ & rs146400809 \\
\hline Chr2: 203395591 & $B M P R 2$ & Nonsyn SNV & NM_001204 & c. $1042 \mathrm{G}>\mathrm{A}$ & rs201067849 \\
\hline Chr3: 38607905 & SCN5A & Nonsyn SNV & NM_000335 & c. $3832 \mathrm{G}>\mathrm{A}$ & rs199473341 \\
\hline Chr3: 38674698 & SCN5A & Nonsyn SNV & NM_000335 & c. $101 \mathrm{G}>\mathrm{A}$ & rs199473046 \\
\hline Chr3: 132438619 & NPHP3 & Nonsyn SNV & NM_153240 & c. $449 \mathrm{C}>\mathrm{T}$ & rs142663818 \\
\hline Chr7: 42064927 & GLI3 & Nonsyn SNV & NM_000168 & c. $1292 \mathrm{~A}>\mathrm{G}$ & - \\
\hline Chr9: 34506694 & DNAI1 & Nonsyn SNV & NM_012144 & c. $1133 \mathrm{~A}>\mathrm{T}$ & - \\
\hline Chr9: 97080945 & $F A M 22 F$ & Del & NM_017561 & c.2071_2073delTCT & rs150455117 \\
\hline Chr11: 1267969 & $M U C 5 B$ & Nonsyn SNV & NM_002458 & c. $9859 \mathrm{~A}>\mathrm{C}$ & - \\
\hline Chr11: 1271591 & $M U C 5 B$ & Nonsyn SNV & NM_002458 & c. $13481 \mathrm{~A}>\mathrm{C}$ & rs201038498 \\
\hline Chr11: 47356616 & MYBPC3 & Unknown & NM_000256 & c. $2883 \mathrm{C}>\mathrm{T}$ & - \\
\hline Chr11: 126143258 & FOXRED1 & Frameshift Del & NM_017547 & c. $445 \mathrm{delC}$ & - \\
\hline Chr12: 58177067 & TSFM & Splicing & NM_001172695 & c. $231+1 \_231+2$ delGT & - \\
\hline Chr12: 112892433 & PTPN11 & Stop-gain & NM_002834 & c. $591 \mathrm{~T}>\mathrm{G}$ & rs76982592 \\
\hline Chr16: 1245007 & CACNA1H & Nonsyn SNV & NM_001005407 & c. $335 \mathrm{~T}>\mathrm{C}$ & - \\
\hline Chr16: 71061495 & HYDIN & Stop-loss & NM_017558 & c. $3052 \mathrm{~T}>\mathrm{C}$ & rs146649547 \\
\hline Chr16: 89815152 & FANCA & Nonsyn SNV & NM_000135 & c. $3263 \mathrm{C}>\mathrm{T}$ & rs17233497 \\
\hline Chr21: 35821734 & KCNE1 & Nonsyn SNV & NM_001127670 & c. $199 \mathrm{C}>\mathrm{T}$ & rs199473645 \\
\hline
\end{tabular}

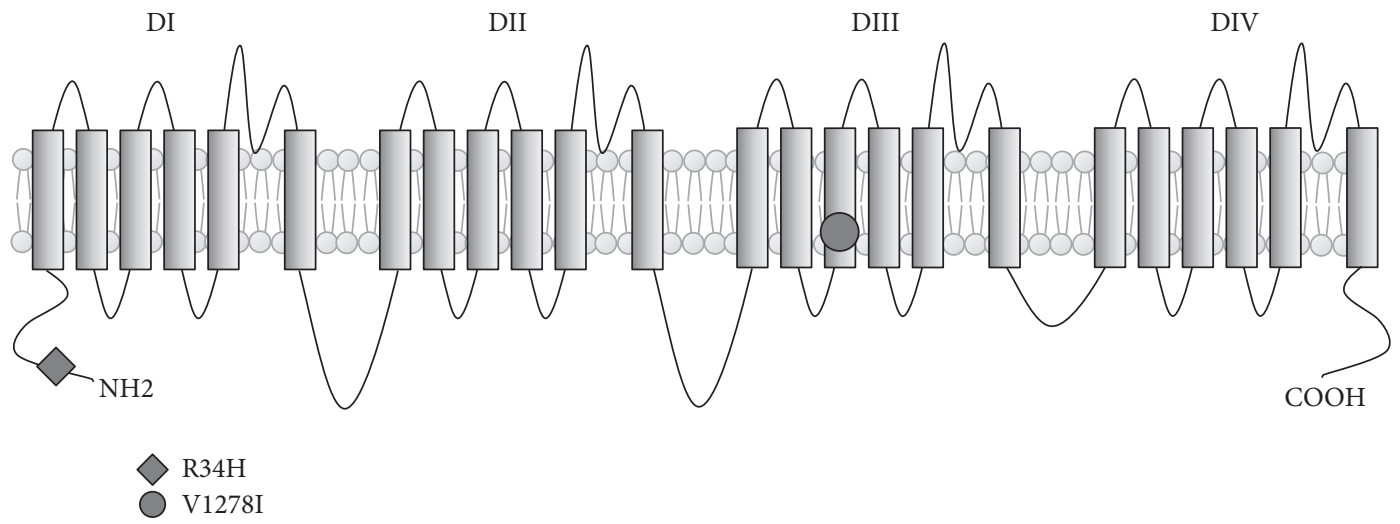

FIGURE 5: Schematic of the transmembrane topology of the SCN5A protein. The location of the variants R34H (c.101G>A) and V1278I (c.3832G $>\mathrm{A}$ ) is shown.

transmembrane segment of domain III (DIII) of the sodium channel alpha subunit. McNair et al. have reported that heterozygous c. $3823 \mathrm{G}>\mathrm{A}$ variant, which is located near c. $3832 \mathrm{G}>\mathrm{A}$, was associated with dilated conduction disorder, cardiomyopathy, and arrhythmia [34]. In the same region, an association between atrial standstill and p.D1275N with polymorphisms in other gap junction protein, connexin 40, has been reported by Groenewegen et al. [35]. Electrophysiological studies in xenopus oocytes showed that the c.3823G>A mutation results in an activation curve shift of the sodium channel conductance [35]. This activation curve shift toward more positive voltages may result in reduced excitability of myocytes. According to studies of Gosselin-Badaroudine et al. and Moreau et al., this electrical disturbance could be the result from positive charge leakages of mutated NaV1.5 channels that could lead to a $\mathrm{Na}+$ leak into cardiac myocytes $[27,28]$.

Results from segregation analysis indicated that both variants in the SCN5A gene were most possibly inherited in a compound heterozygous manner. Heterozygous c.101G $>$ A was present in family members III-2 (index) and II-2 but was absent in family members I-2 and III-3. Likewise, heterozygous c.3832G $>$ A was present in family members III2 (index) and III-3 but was absent in family members I-2 and II-2 while only III- 2 was the heart block affected family member. Although most of reported variants in the SCN5A 


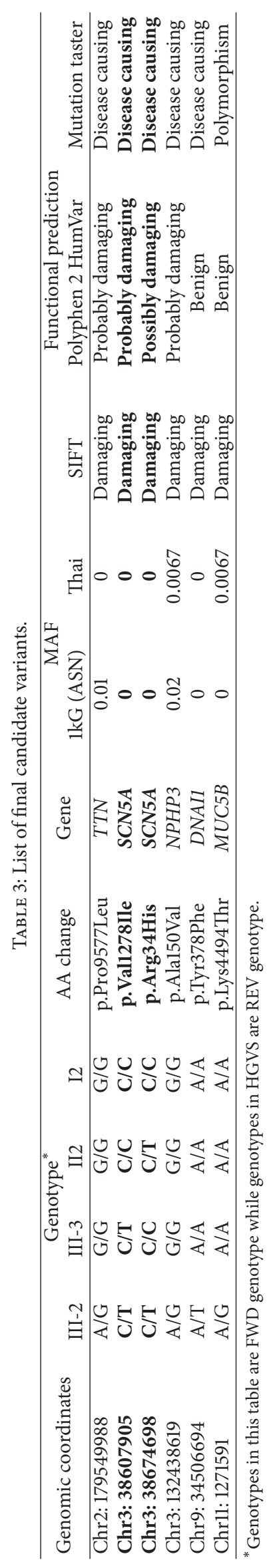


gene were autosomal dominant, however, variants that were inherited in compound heterozygous individuals, similar to the current case finding, have been previously reported.

For instance, Bezzina et al. described compound heterozygous inheritance of 2 variants in the SCN5A gene which was associated with severe cardiac conduction disturbances and degenerative changes in the conduction system [36]. A nonsense p.W156X, which is located in the S1-S2 linker of domain I, was inherited from the father and a missense p.R225W, which was located in S4 segment of domain I, was inherited from the mother [36].

Benson et al. studied compound heterozygous variants in SCN5A in three families with congenital sick sinus syndrome [13]. A heterozygous missense p. P1298L, which is located in S4 segment of domain III, and p.G1408R, which is located in S5-S6 linker of domain III, were found in three siblings of the first family. Heterozygous missense p.T220I and p.R1623X were found in an individual of the second family. These two variants were located in the S4 segment of domain I and S4 segment of domain IV. The third family presented heterozygous deletions p.delF1617 in S3-S4 linker of domain IV and the missense p. R1632H in S4 segment of domain IV [13].

Beside the variants in SCN5A gene, 4 heterozygous missense variants in TTN, NPHP3, DNAI1, and MUC5B genes were found in the present case. All variants were found in only the index case, family member III-2. Among these 4 genes, TTN was the most likely cardiac disease associated gene while lack of evidence for NPHP3, DNAI1, and MUC5B genes existed. Mutations in TTN have been reported in about $18 \%$ of sporadic dilated cardiomyopathies and $25 \%$ of familial autosomal dominant cardiomyopathies and rarely caused hypertrophic cardiomyopathies [37].

Since this study only focused on the coding variants in the exon by whole sequencing, noncoding variants and structural variants were not explored. Moreover, it should be noted that novel variants in the study were found by the bioinformatics process so determination of biological functions will be needed in further studies.

\section{Conclusion}

In conclusion, this study demonstrated the potential of whole exome sequencing and a bioinformatics pipeline to identify the possible causative variants of complete heart block in a Thai family. The investigation found compound heterozygous variants in the SCN5A, cardiac sodium channel subunit gene, of which one was a novel variant and another one was a known pathogenic variant. Additionally, a heterozygous missense variant in the TTN, titin or connectin gene, has also been identified.

\section{Competing Interests}

The authors have no relevant affiliations or financial involvements with any organization or entity with a financial interest in or financial conflict with the subject matter or materials discussed in the manuscript.

\section{Acknowledgments}

This study was supported by grants of the (1) Pharmacogenomics for Autistic Child Project, Khoon Poom Foundation, The Project in Her Royal Highness Princess Ubonratana Rajakanya Siriwatana Bhanawadee, (2) Office of National Research Council of Thailand, (3) Faculty of Medicine Ramathibodi Hospital, (4) Excellence Center for Genomic Medicine, Faculty of Medicine Ramathibodi Hospital, Mahidol University, Bangkok, Thailand, and (5) The Thailand Centre of Excellence for Life Sciences (TCELS). This study was also partially supported by National Center for Genetic Engineering and Biotechnology (BIOTEC), Grant no. BTB-01-MG-18-4814, and the Faculty of Medicine, Prince of Songkla University (48/364-006-3). The authors thank all the staffs in Laboratory for Pharmacogenomics Somdech Phra Debaratana Medical Center (SDMC), Ramathibodi Hospital, Human Genetics Unit, Department of Pathology, Faculty of Medicine, Prince of Songkla University.

\section{References}

[1] D. M. Friedman, L. J. Duncanson, J. Glickstein, and J. P. Buyon, "A review of congenital heart block," Images in Paediatric Cardiology, vol. 5, no. 3, pp. 36-48, 2003.

[2] J. P. Smits, M. W. Veldkamp, and A. A. Wilde, "Mechanisms of inherited cardiac conduction disease," Europace, vol. 7, no. 2, pp. 122-137, 2005.

[3] P. J. Schwartz, A. Garson Jr., T. Paul et al., "Guidelines for the interpretation of the neonatal electrocardiogram: a Task Force of the European Society of Cardiology," European Heart Journal, vol. 23, no. 17, pp. 1329-1344, 2002.

[4] D. S. Park and G. I. Fishman, "The cardiac conduction system," Circulation, vol. 123, no. 8, pp. 904-915, 2011.

[5] K. Ueda, K. Nakamura, T. Hayashi et al., "Functional characterization of a trafficking-defective HCN4 mutation, D553N, associated with cardiac arrhythmia," The Journal of Biological Chemistry, vol. 279, no. 26, pp. 27194-27198, 2004.

[6] P. Y. Jay, B. S. Harris, A. Buerger et al., "Function follows form: Cardiac conduction system defects in Nkx2-5 mutation," Anatomical Record-Part A: Discoveries in Molecular, Cellular, and Evolutionary Biology, vol. 280, no. 2, pp. 966-972, 2004.

[7] I. P. G. Moskowitz, A. Pizard, V. V. Patel et al., "The TBox transcription factor Tbx 5 is required for the patterning and maturation of the murine cardiac conduction system," Development, vol. 131, no. 16, pp. 4107-4116, 2004.

[8] S. Le Scouarnec, N. Bhasin, C. Vieyres et al., "Dysfunction in ankyrin-B-dependent ion channel and transporter targeting causes human sinus node disease," Proceedings of the National Academy of Sciences of the United States of America, vol. 105, no. 40, pp. 15617-15622, 2008.

[9] E. Arbustini, A. Pilotto, A. Repetto et al., "Autosomal dominant dilated cardiomyopathy with atrioventricular block: a lamin A/C defect-related disease," Journal of the American College of Cardiology, vol. 39, no. 6, pp. 981-990, 2002.

[10] C. A. Remme, A. A. M. Wilde, and C. R. Bezzina, "Cardiac sodium channel overlap syndromes: different faces of SCN5A mutations," Trends in Cardiovascular Medicine, vol. 18, no. 3, pp. 78-87, 2008. 
[11] A. L. George Jr., "Inherited disorders of voltage-gated sodium channels," Journal of Clinical Investigation, vol. 115, no. 8, pp. 1990-1999, 2005.

[12] J. J. Schott, C. Alshinawi, F. Kyndt et al., "Cardiac conduction defects associate with mutations in SCN5A," Nature Genetics, vol. 23, no. 1, pp. 20-21, 1999.

[13] D. W. Benson, D. W. Wang, M. Dyment et al., "Congenital sick sinus syndrome caused by recessive mutations in the cardiac sodium channel gene (SCN5A)," Journal of Clinical Investigation, vol. 112, no. 7, pp. 1019-1028, 2003.

[14] C. Bezzina, M. W. Veldkamp, M. P. Van Den Berg et al., "A single $\mathrm{Na}+$ channel mutation causing both long-QT and Brugada syndromes," Circulation Research, vol. 85, no. 12, pp. 1206-1213, 1999.

[15] C. A. Remme, A. O. Verkerk, D. Nuyens et al., "Overlap syndrome of cardiac sodium channel disease in mice carrying the equivalent mutation of human SCN5A-1795insD," Circulation, vol. 114, no. 24, pp. 2584-2594, 2006.

[16] Q. Wang, J. Shen, I. Splawski et al., "SCN5A mutations associated with an inherited cardiac arrhythmia, long QT syndrome," Cell, vol. 80, no. 5, pp. 805-811, 1995.

[17] M. Choi, U. I. Scholl, W. Ji et al., "Genetic diagnosis by whole exome capture and massively parallel DNA sequencing," Proceedings of the National Academy of Sciences of the United States of America, vol. 106, no. 45, pp. 19096-19101, 2009.

[18] M. J. Bamshad, S. B. Ng, A. W. Bigham et al., "Exome sequencing as a tool for Mendelian disease gene discovery," Nature Reviews Genetics, vol. 12, no. 11, pp. 745-755, 2011.

[19] S. C. Greenway, R. McLeod, S. Hume et al., "Exome sequencing identifies a novel variant in ACTC1 associated with familial atrial septal defect," Canadian Journal of Cardiology, vol. 30, no. 2, pp. 181-187, 2014.

[20] C. Genomes Project, G. R. Abecasis, A. Auton et al., "An integrated map of genetic variation from 1,092 human genomes," Nature, vol. 491, no. 7422, pp. 56-65, 2012.

[21] X. Liu, X. Jian, and E. Boerwinkle, "dbNSFP v2.0: a database of human non-synonymous SNVs and their functional predictions and annotations," Human Mutation, vol. 34, no. 9, pp. E2393-E2402, 2013.

[22] R. C. Green, J. S. Berg, W. W. Grody et al., "ACMG recommendations for reporting of incidental findings in clinical exome and genome sequencing," Genetics in Medicine, vol. 15, no. 7, pp. 565-574, 2013.

[23] P. D. Stenson, M. Mort, E. V. Ball, K. Shaw, A. D. Phillips, and D. N. Cooper, "The human gene mutation database: building a comprehensive mutation repository for clinical and molecular genetics, diagnostic testing and personalized genomic medicine," Human Genetics, vol. 133, no. 1, pp. 1-9, 2014.

[24] A. Untergasser, I. Cutcutache, T. Koressaar et al., "Primer3-new capabilities and interfaces," Nucleic Acids Research, vol. 40, no. 15, article e115, 2012.

[25] I. Splawski, K. W. Timothy, N. Decher et al., "Severe arrhythmia disorder caused by cardiac L-type calcium channel mutations," Proceedings of the National Academy of Sciences of the United States of America, vol. 102, no. 23, pp. 8089-8096, 2005.

[26] T. Zimmer and R. Surber, "SCN5A channelopathies-an update on mutations and mechanisms," Progress in Biophysics and Molecular Biology, vol. 98, no. 2-3, pp. 120-136, 2008.

[27] P. Gosselin-Badaroudine, D. I. Keller, H. Huang et al., "A proton leak current through the cardiac sodium channel is linked to mixed arrhythmia and the dilated cardiomyopathy phenotype," PLoS ONE, vol. 7, no. 5, Article ID e38331, 2012.
[28] A. Moreau, P. Gosselin-Badaroudine, L. Delemotte, M. L. Klein, and M. Chahine, "Gating pore currents are defects in common with two Nav1.5 mutations in patients with mixed arrhythmias and dilated cardiomyopathy," The Journal of General Physiology, vol. 145, no. 2, pp. 93-106, 2015.

[29] S. G. Priori, C. Napolitano, M. Gasparini et al., "Natural history of Brugada syndrome: insights for risk stratification and management," Circulation, vol. 105, no. 11, pp. 1342-1347, 2002.

[30] N. Makita, N. Sumitomo, I. Watanabe, and H. Tsutsui, "Novel SCN5A mutation (Q55X) associated with age-dependent expression of Brugada syndrome presenting as neurally mediated syncope," Heart Rhythm, vol. 4, no. 4, pp. 516-519, 2007.

[31] W. P. McNair, G. Sinagra, M. R. G. Taylor et al., "SCN5A mutations associate with arrhythmic dilated cardiomyopathy and commonly localize to the voltage-sensing mechanism," Journal of the American College of Cardiology, vol. 57, no. 21, pp. 2160-2168, 2011.

[32] L. M. Amendola, M. O. Dorschner, P. D. Robertson et al., "Actionable exomic incidental findings in 6503 participants: challenges of variant classification," Genome Research, vol. 25, no. 3, pp. 305-315, 2015.

[33] C. Andreasen, J. B. Nielsen, L. Refsgaard et al., "New population-based exome data are questioning the pathogenicity of previously cardiomyopathy-associated genetic variants," European Journal of Human Genetics, vol. 21, no. 9, pp. 918-928, 2013.

[34] W. P. McNair, L. Ku, M. R. G. Taylor et al., "SCN5A mutation associated with dilated cardiomyopathy, conduction disorder, and arrhythmia," Circulation, vol. 110, no. 15, pp. 2163-2167, 2004.

[35] W. A. Groenewegen, M. Firouzi, C. R. Bezzina et al., "A cardiac sodium channel mutation cosegregates with a rare connexin 40 genotype in familial atrial standstill," Circulation Research, vol. 92, no. 1, pp. 14-22, 2003.

[36] C. R. Bezzina, M. B. Rook, W. A. Groenewegen et al., "Compound heterozygosity for mutations (W156X and R225W) in SCN5A associated with severe cardiac conduction disturbances and degenerative changes in the conduction system," Circulation Research, vol. 92, no. 2, pp. 159-168, 2003.

[37] D. S. Herman, L. Lam, M. R. G. Taylor et al., "Truncations of titin causing dilated cardiomyopathy," The New England Journal of Medicine, vol. 366, no. 7, pp. 619-628, 2012. 


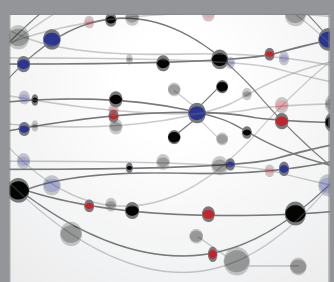

The Scientific World Journal
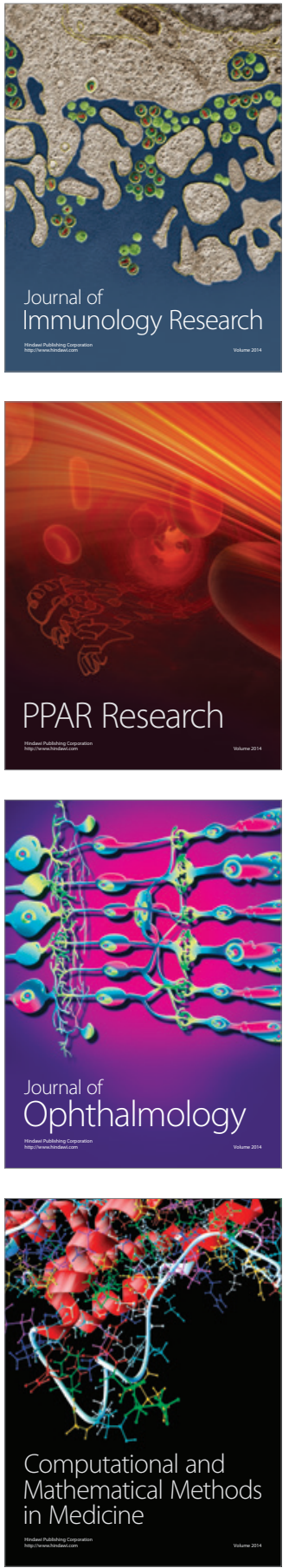

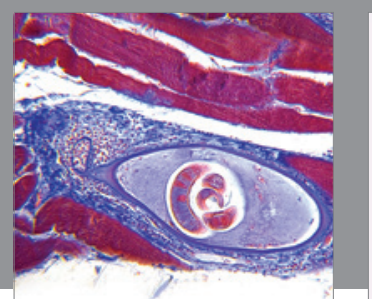

Gastroenterology Research and Practice

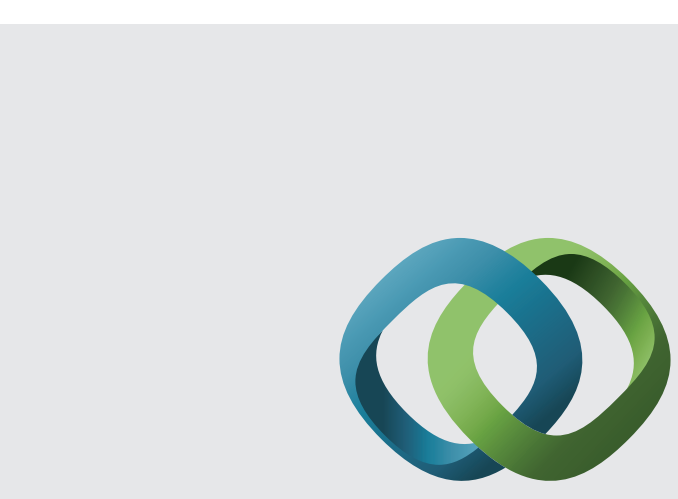

\section{Hindawi}

Submit your manuscripts at

http://www.hindawi.com
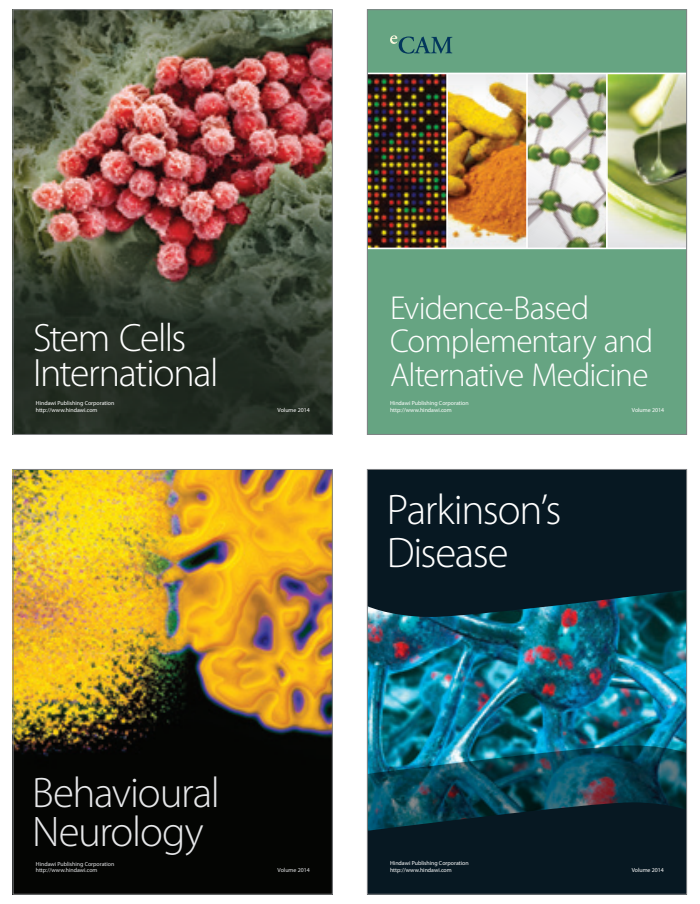
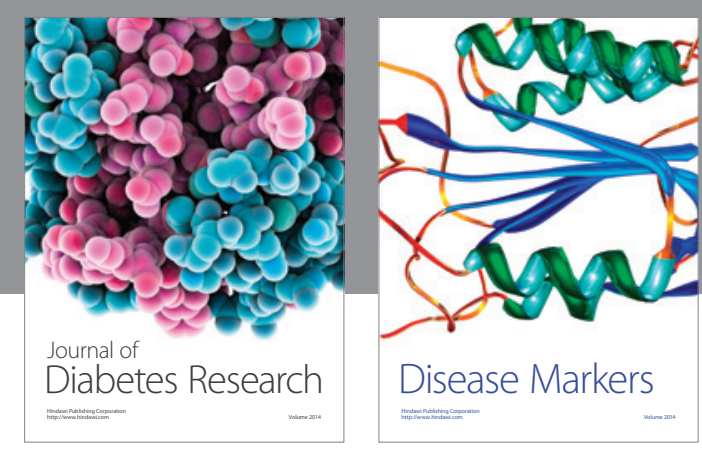

Disease Markers
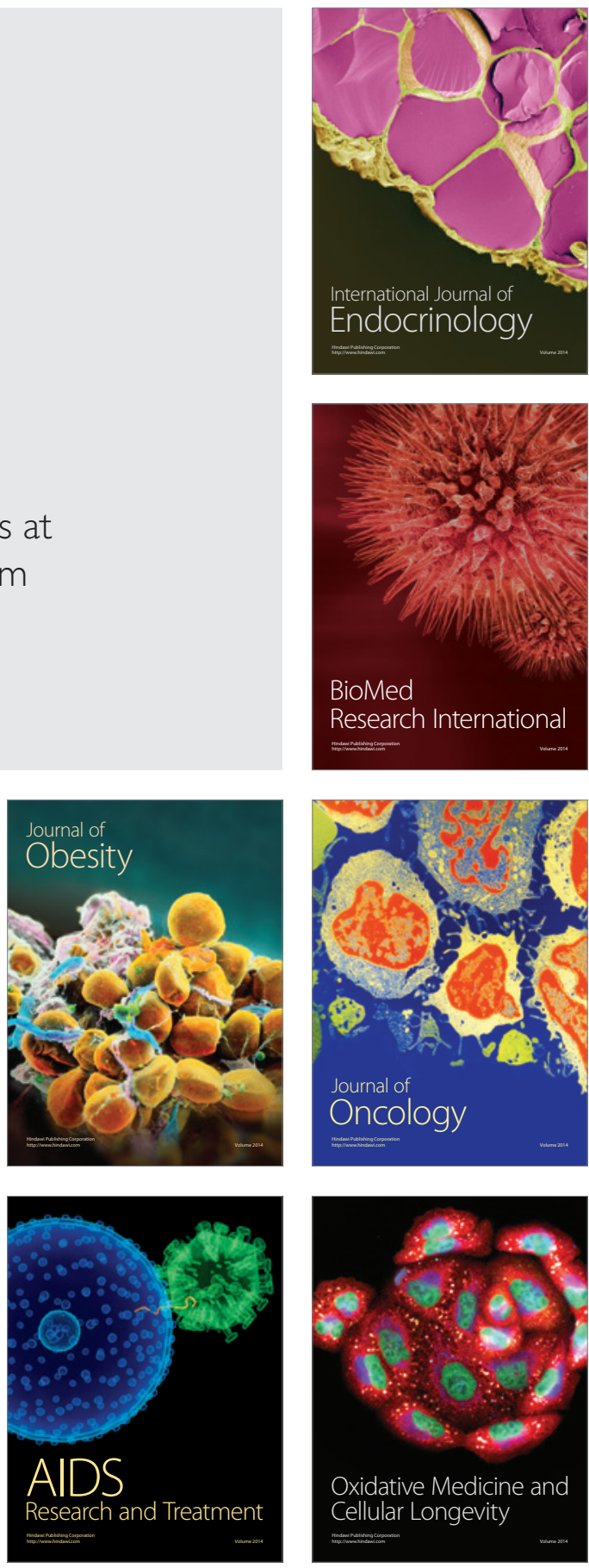